\title{
LA FUERZA EN LA CONSTRUCCIÓN DEL MARCO TEÓRICO EN ARTÍCULOS CIENTÍFICOS: UN ANÁLISIS DESDE LA LINGÜÍSTICA SISTÉMICO-FUNCIONAL
}

\author{
Force in the Construction of the Theoretical Framework in Scientific Papers: \\ A Systemic-Functional Linguistics Approach \\ LA FORCE DANS LA CONSTRUCTION DU CADRE THÉORIQUE DANS DES ARTICLES SCIENTIFIQUES : \\ UNE ANALYSE PARTANT DE LA LINGUISTIQUE SYSTÉMIQUE-FONCTIONNELLE
}

\author{
Norma Patricia Barletta \\ Ph. D. Second Language Acquisition \\ and Teaching, Universidad de Arizona, \\ EE.UU. \\ Profesora asociada, Departamento \\ de Español, Universidad del Norte, \\ Colombia. \\ Km. 5, vía Puerto Colombia, Barran- \\ quilla, Colombia. \\ nbarlett@uninorte.edu.co \\ https://orcid. \\ org/0000-0003-0180-5620
}

\section{Diana Judith Chamorro}

Doctora en Métodos de investigación, Universidad Complutense, Madrid, España.

Profesora asociada, Departamento de Español, Universidad del Norte, Colombia.

Km. 5, vía Puerto Colombia, Barranquilla, Colombia.

dchamorro@uninorte.edu.co

https://orcid.

org/0000-0001-7928-2861

\section{Jorge Mizuno}

Magíster Educación, Universidad del Norte, Colombia.

Profesor emérito, Universidad del

Norte, Colombia.

\begin{abstract}
RESUMEN
Contrario a lo que se ha señalado tradicionalmente, el marco teórico muchas veces constituye una sección independiente de la introducción en los artículos de investigación. En este, los escritores establecen la tradición teórica desde la cual se desarrolla la investigación y hacen un recuento de los avances teóricos e investigativos en la disciplina y específicamente en el tema del estudio, estableciendo un diálogo con otras voces. En este artículo, se examina cómo los escritores organizan su marco teórico mediante las cláusulas verbales y los recursos del subsistema de graduación propuestos por la lingüística sistémico-funcional para expresar fuerza y construir su voz autorial. Se identificaron las cláusulas verbales y los recursos del subsistema de graduación asociados a ellas en las secciones de marco teórico de veinte artículos científicos en español, publicados en revistas colombianas del área de la lingüística aplicada. El análisis mostró la variedad de recursos con los que los escritores dan fuerza a sus referentes conceptuales, desde las cláusulas verbales, especialmente en lugares prominentes del marco teórico: en los macroTemas, hiperTemas, macroNuevos e hiperNuevos. Comprender estas características del marco teórico puede ser relevante para la enseñanza de la escritura académica.
\end{abstract}

Palabras clave: artículo de investigación; fuerza; graduación; lingüística sistémico-funcional; marco teórico; artículo científico.

\section{Abstract}

Opposite to what is traditionally stated, the theoretical framework often constitutes an independent section in research articles. Therein writers establish the theoretical tradition that supports their research and make an account of the theoretical and research developments in their discipline, specifically, in their topic of research, thus establishing a dialogue with other voices. This article examines how writers organize their theoretical framework by means of verbal clauses using the resources of the graduation subsystem proposed by the systemic functional linguistics to express force - which will help them to construct their authorial voice. This study analyzed the verbal clauses 
Km. 5, vía Puerto Colombia, Barranquilla, Colombia.

jmizzuno@uninorte.edu.co

https://orcid.

org/0000-0002-5083-3540
Este artículo se deriva del estudio "El uso de los procesos verbales en español para expresar valoración en textos científicos en el área de lenguaje", llevado a cabo en la Universidad del Norte, Barranquilla, Colombia entre 2014 a 2018 como parte del proyecto internacional SAL (Systemics Across Languages). El proyecto SAL está coordinado por Christian Matthiessen y Kazuhiro Teruya, de la Universidad Politécnica de Hong Kong, y por Leila Barbara, de la Universidad Católica de São Paulo, Brasil. En el proyecto participan también universidades de China, Japón, Tailandia, Argentina, Brasil, Colombia y México. and the graduation resources of the theoretical frameworks in 20 research articles in Spanish language published in Colombian journals from the area of applied linguistics. Findings show the variety of resources deployed by writers in their verbal clauses to strengthen their commitment to their tenets, especially in prominent places of their theoretical framework, such as the macroTheme, hyperThemes, macroNew and hyperNew. Awareness of these theoretical framework features may be relevant for teaching academic writing.

Keywords: research article; force; graduation; systemic-functional linguistics; theoretical framework; scientific paper.

\section{RÉSUMÉ}

Contrairement à ce qui a été traditionnellement dit, le cadre théorique constitue souvent une section indépendante de l'introduction dans les articles de recherche. Dans le cadre théorique, les auteurs élaborent la tradition théorique à partir de laquelle leur recherche est développée et font une recension de l'histoire des avancées de la théorie et de la recherche dans leur discipline et, plus particulièrement, selon leur sujet d'étude. Il s'y établit ainsi une polyphonie conceptuelle. Dans cet article, nous examinons comment les auteurs organisent leur cadre théorique en ayant recours à des propositions verbales et en s' appuyant sur les ressources du sous-système de la gradation de la linguistique systémique fonctionnelle pour exprimer de la solidité théorique et ainsi construire leur voix auctoriale. Nous avons analysé les propositions verbales ainsi que les ressources du sous-système de gradation associées dans la section du cadre théorique de vingt articles scientifiques en espagnol, publiés dans des revues spécialisées du domaine de la linguistique appliquée. L'analyse a montré la diversité des ressources déployées par les auteurs visant à donner de la force à leurs référents conceptuels à partir des propositions verbales, en particulier à des endroits bien précis dans le cadre théorique : dans les macro-thèmes, hyper-thèmes, macro-nouveaux et hyper-nouveaux. La compréhension de ces caractéristiques du cadre théorique peut être pertinente pour l'enseignement de l'écriture universitaire.

Mots clés : article de recherche; force; gradation ; linguistique systémique-fonctionnelle; cadre théorique; article scientifique. 


\section{Introducción}

El artículo de investigación es uno de los géneros más utilizados en el ámbito académico, pues da cuenta de los avances en los procesos de construcción de conocimiento en los diferentes campos de la ciencia. En este contexto, la construcción de identidad por parte del escritor es un aspecto fundamental para posicionarse como investigador, experto y miembro de una comunidad académica. Para ello, resulta importante movilizar algunos recursos que realicen la función interpersonal del lenguaje y den cuenta del tipo de actitud (positiva o negativa) del escritor hacia otros autores y fenómenos, la fuerza de esa evaluación, y el compromiso con otras voces, es decir, si se suprime o se abre el diálogo hacia ellas o, en otras palabras, si se tienen o no en cuenta esas otras voces. Esta naturaleza dialógica del discurso puede ser implícita, cuando el texto no reconoce abiertamente la existencia de otros puntos de vista, en cuyo caso el discurso es monoglósico, pero cuando el discurso hace explícita su naturaleza dialógica, se trata de un discurso heteroglósico. Estos significados son realizados mediante el sistema de la VALORACión (Hood y Martin, 2005; Martin y White, 2005).

Son de especial interés en el estudio que aquí se presenta los recursos que construyen la FUERZA en las aseveraciones en los artículos científicos, específicamente en el marco teórico (en adelante, MT), mediante el subsistema de la GRADUACIÓN. Los estudios de GRADUACIÓn, por lo general, han estado asociados a los de COMPROMIso. En inglés, estos dos sistemas se han investigado en géneros como: avisos publicitarios (Beangstrom y Adendorff, 2013); noticias, editoriales, cartas al editor (Santosa, Priyanto y Nuraeni 2014); tweets (Zappavinga, 2011); diatribas, rallies y reflexiones en las redes sociales (Sutherland y Adendorff, 2014); tesis doctorales (ChatterjeePadmanabhan, 2011; Xiaoyu, 2017); discusiones entre docentes (Love y Arkoudis, 2006), y en el desarrollo de la expresión de la función interpersonal en la escritura de adolescentes (Derewianka, 2007).
En español, se ha analizado la GRADUACIÓN en sentencias judiciales (Cunillera-Domènech y Andújar-Moreno, 2017; Serpa, 2013), en artículos de investigación en ciencias económicas (Marimón, 2014), en el tratamiento de derechos humanos en la prensa argentina (Pascual, 2014), en exámenes presenciales (Navarro, 2014) y en presentaciones orales (Cisneros-Estupiñán y Muñoz, 2014), pero no encontramos estudios en los que se examine la GRADUACIÓN en el MT, en donde las cláusulas verbales cumplen un papel importante. En esa sección, es necesario hacer referencia a los trabajos publicados por otros investigadores (Hood, 2010; Ignatieva, 2011; Martin y White, 2005; Moyano, 2010) y muchas veces esto se hace mediante cláusulas del decir.

Cada comunidad académica y disciplinar desarrolla sus propias prácticas para la generación de conocimiento y sistemas específicos de legitimación de dichas prácticas (Maton, 2007). A este respecto, Bazerman (1988, p. 20) afirma: "cada comunidad al establecer su propia manera de formular el conocimiento define qué es el conocimiento de la comunidad". 'Así, serán aceptados como generadores de conocimiento aquellos investigadores que aprendan a utilizar el discurso de su comunidad para comunicar sus ideas. Quienes no logren producir el discurso aceptado en su comunidad quedarán marginados.

Nos proponemos entonces utilizar algunas de las herramientas analíticas propuestas por la lingüística sistémico-funcional (LSF) para realizar un análisis de cómo se realiza la FUERZA en las cláusulas verbales en el MT de artículos científicos publicados en revistas colombianas del área del lenguaje. De esta manera, queremos contribuir a la comprensión de cómo se construye el MT y cómo los escritores se posicionan en la comunidad científica, utilizandolas voces de otros autores para justificar la selección de las teorías utilizadas para sus estudios e imprimir mayor validez a sus investigaciones.

1 Esta traducción y las siguientes son de los autores de este artículo. 
El corpus lo constituyen los MT de veinte artículos de investigación publicados en cuatro revistas colombianas indexadas del área de lingüística aplicada. En ellos, se analizó la manera como se expresa la FUERZA en las cláusulas verbales. Inicialmente, definiremos los conceptos centrales del subsistema de FUERZA dentro del sistema de GRADUACIÓN de la LSF, así como la noción de periodicidad o flujo de la información en los textos. Seguidamente, describiremos la metodología utilizada, los resultados encontrados y las conclusiones del estudio.

\section{Marco teórico}

Según Hood (2010), en los textos académicos, cuando los autores reportan otras investigaciones, se despliegan diversos recursos para invocar la ACTITUD del lector o lectora con respecto a la contribución de otros estudios o autores. En el sistema de la VALORACión, Martiny White (2005) identifican dos maneras como se puede graduar la ACTITUD: FUERZA y FOCO. La primera se refiere a los recursos para ajustar el grado, la cantidad o la intensidad de la evaluación, para subir o bajar su volumen (Kaplan, 2004; Martin y White, 2005). La segunda opera en el contexto de los recursos que no son graduables; se refiere a los recursos para ajustar o delimitar las fronteras entre categorías, y construir así tipos de entidades que son más centrales o periféricas, más o menos prototípicas (Martin y White, 2005). Los recursos de estos sistemas, que tienen una función dialógica, permiten a los escritores presentarse como más o menos alineados con las posiciones que adoptan en un texto o ubicarse con respecto a una comunidad que comparte los valores de estas posiciones. En este artículo, nos ocuparemos de la manera como los autores intensifican o disminuyen la intensidad y la cantidad de la FUERZA que imprimen a sus posturas al emplear cláusulas verbales.

\section{Fuerza}

Martin y White (2005) establecen que la FUERZA puede expresarse como intensificación o cuantificación. La intensificación se refiere a la evaluación del grado de intensidad de cualidades o procesos, mientras que la cuantificación es el escalamiento de la cantidad y la extensión de las entidades y los procesos. En ambos casos, se construye una orientación subjetiva, cuya polaridad se deduce de la interpretación del cotexto. Estas categorías de FUERZA, que adaptamos de Hood (2010) y Martin y White (2005), se relacionan en la Figura 1.

\section{Prosodia, propagación y acoplamiento}

Los significados interpersonales se construyen mediante estructuras prosódicas que se realizan a través de la cláusula con efecto acumulativo, por medio de fragmentos continuos de discurso y le imprimen un tono o un cierto colorido (Halliday, 1979). La prosodia se comprende como la extensión o difusión de los significados interpersonales que, al acumularse, refuerzan o tiñen un segmento con un tono evaluativo particular (Halliday y Matthiessen, 1999,

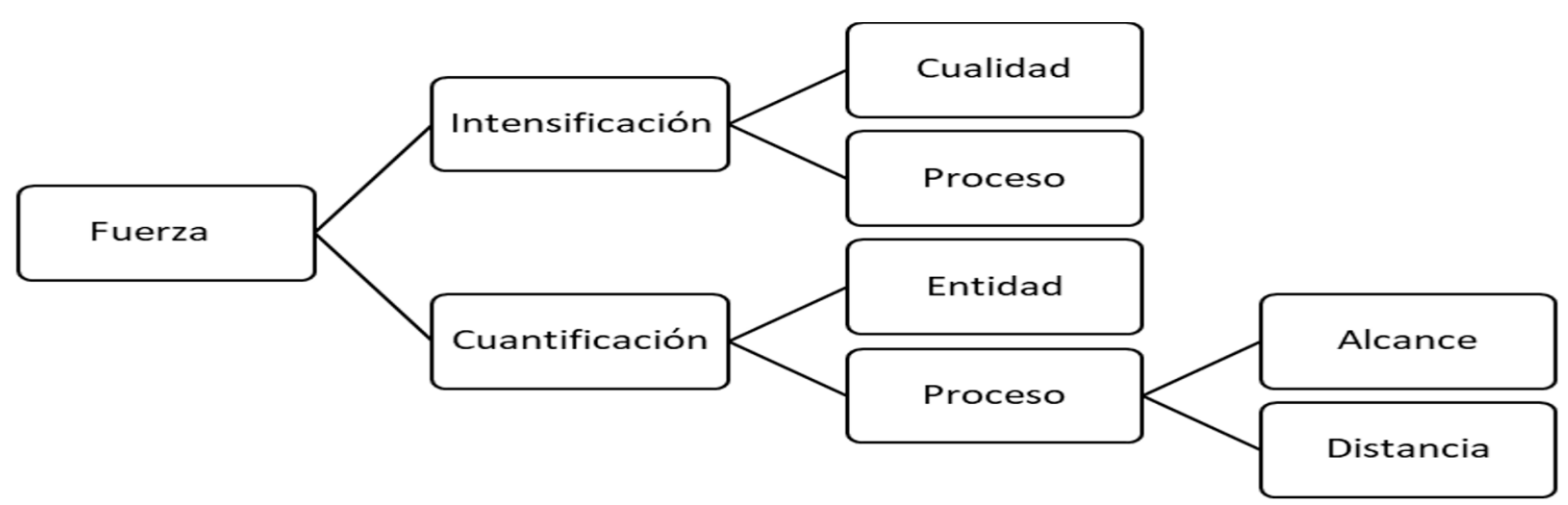

Figura 1 Categorías de FUERZA (adaptado de Hood, 2010; Martin y White, 2005) 
Hood, 2010; Martin 1992; Martin y Rose, 2007; Thompson, 1998).

Estas prosodias se extienden a otros segmentos o fases mediante el proceso de propagación (Hood, 2010). Asimismo, resulta particularmente útil el recurso de acoplamiento (coupling), que alude a "la forma como los significados se combinan —a través de estratos, metafunciones, rasgos, y sistemas simultáneos" (Martin, 2010, p. 19), y a la forma como un significado ideacional se carga con ACTITUD positiva/negativa inscrita en otro elemento, en la misma cláusula/fase; también ocurre cuando elementos ideacionales asumen significados actitudinales por influencia del significado actitudinal adquirido por otro significado ideacional.

Según Hood (2010) y Martin y White (2005), la prosodia puede realizarse mediante los mecanismos de dominación, intensificación y saturación, los cuales ocurren por propagación, a nivel de cláusula, en conexiones gramaticales; en relaciones de proyección en un complejo de cláusulas; en enlaces cohesivos (cantidad o extensión), y en fases más largas de texto (Hood, 2006; Lemke, 1998). Para el caso de la FUERZA, la prosodia se da por dominación y saturación, puesto que la intensificación es una forma de FUERZA (ver sección Contrucción prosódica de la FUERZA). Tal como lo plantea Hood (2010), "las prosodias de dominación son comunes en fases que reportan sobre el objeto de estudio y en las que reportan otras investigaciones. Las prosodias de saturación son más comunes cuando el escritor reporta el objeto de estudio" (p. 157). En el MT, entonces, se espera identificar prosodias de dominación, construidas de forma prospectiva o retrospectiva.

Hood (2006) asigna un papel importante a la interacción entre GRADUACIÓN y ACTITUD para mantener prosodias de valor en las introducciones de los artículos de investigación. Hood parte de que las introducciones utilizan tres estrategias principales: mostrar la importancia y relevancia del tema, que amerita su estudio; encontrar aspectos no estudiados suficientemente, y valorar positivamente el estudio propio y su contribución. El uso de estas estrategias supone poner en juego significados interpersonales. En los datos de Hood, un emisor a quien se le otorga alta valoración aumenta el valor atribuido a lo proyectado, cuando el significado del proceso tiene una carga de GRADUACIÓN. Así, por ejemplo, un proceso como 'x demuestra', es percibido con mayor intensidad que ' $x$ sugiere'. Asimismo, una proyección con un valor positivo puede, retrospectivamente, propagar este valor al emisor.

En este artículo se muestra cómo los procesos verbales no solamente realizan significados experienciales, sino también significados interpersonales de GRADUACIÓN de intensificación y cuantificación, mediante procesos infundidos (explícitamente evaluativos) o como punto de gravitación o de condensación de una serie de recursos que escalan la valoración: cualidades, cuantificadores, acoplamiento y prosodia.

\section{Periodicidad o flujo de la información}

En la LSF, la metafunción textual alude a la función del lenguaje encargada de organizar el mensaje. Se cumple mediante patrones que se despliegan en lugares prominentes; por ejemplo, el párrafo introductorio es el nivel máximo de un texto - el macroTema-, cuya función principal es presentar el campo del texto. En cada párrafo, los hiperTemas son las oraciones tópico o picos secundarios, cuya función es introducir el campo de cada párrafo o fase, anunciar qué tipo de información sigue, mientras que el hiperNuevo tiene la función de dar cuenta de lo que se ha presentado antes en el párrafo o en la fase (Rose y Martin, 2012, p, 273), y el macroNuevo recoge lo dicho en el texto o sección.

\section{El marco teórico}

Típicamente se ha considerado que el artículo de investigación tiene una estructura estable: introducción, metodología, resultados y discusión (Swales, 1990). La introducción, según Swales (1990), consta de tres etapas (movimientos): "establecer 
el territorio", "establecer el nicho de la investigación” y "anunciar la investigación”. Para establecer el territorio de la investigación, los escritores pueden recurrir a fases para marcar la importancia del tema, hacer generalizaciones, discutir las investigaciones previas relevantes (Swales, 1990) y hacer inferencias de estudios previos (Pho, 2013). En ese movimiento, Swales (2004) considera obligatoria la referencia a otros autores.

La etapa de establecimiento del territorio en la introducción guarda mucha similitud con la sección del MT, que, cuando se encuentra en el artículo de investigación, está localizado entre las secciones introducción y metodología. El MT, según Tseng (2018), puede considerarse un complemento al territorio establecido en la Introducción, pero en lugar de centrarse en el nicho, se centra en la teoría, e incluso contiene sus propias etapas (o movimientos): "ofrecer un antecedente teórico", "establecer un marco conceptual” y "realzar el significado o el foco del estudio que usa el marco".

En consecuencia, en el MT es importante mostrar que se está construyendo conocimiento dentro de una tradición en un área específica, a cuya elaboración o aplicación han contribuido varios autores, y en la cual se pretende hacer un avance; allí se anuncia un concepto, una teoría o una perspectiva; se declara su importancia, y se presentan o comentan uno o varios trabajos seminales o representativos de la perspectiva adoptada. Todo esto se relaciona con el tema que se presenta en el artículo, en donde se recontextualiza la teoría o el concepto en un problema específico y se reafirma la capacidad para abordarlo.

Es importante anotar que en la naturaleza de esta sección se presenta una especie de contradicción, puesto que de los escritores se espera objetividad $y$, al mismo tiempo, criticidad con respecto a lo reportado o aseverado (Hood, 2010). Esta contradicción se resuelve cuando el escritor reporta las teorías que ha utilizado para realizar su investigación (función experiencial del lenguaje), pero al mismo tiempo recurre a otras voces (Hood,
2010; Martin y White, 2005) con las que se establece un diálogo, que puede ser de aceptación, rechazo, justificación, es decir, un posicionamiento con respecto a lo reportado (función interpersonal del lenguaje). Así, el marco teórico trasciende la noción de "bibliografía anotada" (Swales y Lindemann, 2002), se constituye en garante de la investigación (Hood, 2010) y permite al escritor construir su voz autorial e insertarse en una comunidad discursiva (Oteíza y Pinuer, 2013).

\section{Procesos verbales}

Los procesos verbales, que constituyen el eje de nuestro estudio, son los verbos del decir. Estos son aquellos procesos que manifiestan una relación simbólica que tiene lugar en la conciencia (Halliday y Matthiessen, 2014). Incluyen: los verbos del decir que pueden tener una relación de proyección con otra cláusula; por ejemplo, decir, expresar, comunicar, incluyendo aquellos que indican (mostrar, demostrar) (Halliday y Matthiessen, 2014); los verbos del decir o comunicar que no proyectan (orar, alabar); los procesos conductuales relacionados con la creación de una representación simbólica (caracterizar, esquematizar, bosquejar, hacer una anotación); los que se encuentran entre lo verbal y lo material, es decir, los de 'impacto verbal' (acusar, culpar, felicitar, y criticar) (Matthiessen, 1995), y los que están entre lo verbal y lo (que tradicionalmente es) conductual (hablar, conversar).

En las cláusulas verbales, los participantes más frecuentes son: el emisor, quien dice algo, aunque puede ser una entidad o una abstracción; el destinatario, o entidad a quien se dirige lo dicho; y el informe (verbiage), lo que se dice o el contenido de lo que se dice. En la Tabla 1 se presentan algunos ejemplos tomados del corpus para ilustrar lo anterior.

La función de estos procesos verbales varía según el tipo de texto. En los narrativos, dichos procesos verbales contribuyen a la creación de pasajes dialógicos; en los informes de noticias, permiten al reportero atribuir información a las fuentes, incluidos funcionarios, expertos y testigos oculares 
Tabla 1 Ejemplos de cláusulas verbales y sus participantes

\begin{tabular}{|c|c|c|}
\hline Emisor & Proceso & Informe \\
\hline Varios & han señalado & la importancia de la \\
\hline estudiosos & & concepción de la norma de la \\
\hline \multirow{8}{*}{ El citado autor } & & cortesía \\
\hline & enfatiza & la necesidad de que el lector \\
\hline & & tome conciencia como sujeto \\
\hline & & activo en la construcción del \\
\hline & Serán abordadas & $\begin{array}{l}\text { sentido del texto } \\
\text { las exigencias implicadas }\end{array}$ \\
\hline & & tanto en la producción de \\
\hline & & estos textos como en el acto \\
\hline & & argumentativo \\
\hline
\end{tabular}

Fuente: Corpus del estudio

(Halliday y Matthiessen, 2014, p. 252); en el discurso académico, permiten no solo citar e informar sobre estudios realizados, sino también indicar la postura del escritor con verbos como señalar, sugerir, reclamar y afirmar (Halliday y Matthiessen, 2014, p. 253). Moyano (2010) identifica, además, las funciones de los procesos verbales en la construcción del campo de la disciplina: referencia a la literatura, confrontación de resultados y literatura, realce de los elementos de la argumentación, interpretación de resultados, reconstrucción de los procesos de investigación y relación de las partes del artículo.

Estudios sobre la distribución de los procesos verbales en español demuestran que la frecuencia de su uso varía de acuerdo con el género (Ignatieva y Rodríguez-Vergara, 2015). Textos de algunos géneros, como la revisión de literatura, pueden incluir un porcentaje elevado de procesos verbales, ya que en estos textos se hace referencia constante a otros autores. En el caso de las revistas especializadas, la investigación de Herrero Rivas (2013) también encuentra un número importante de procesos verbales en un corpus de artículos del área de lingüística aplicada.

\section{Método}

Los resultados que presentamos en este artículo se derivan del estudio "El uso de los procesos verbales en español para expresar valoración en textos científicos en el área de lenguaje”, llevado a cabo en la Universidad del Norte, Barranquilla, Colombia, como parte del proyecto internacional SAL (Systemics Across Languages). Uno de los objetivos fue determinar cómo se realiza la FUERZA en las cláusulas verbales en el MT de artículos científicos. Para ello utilizamos las categorías del sistema de la VALORACIÓN de la LSF (Martin y White, 2005) en los marcos teóricos de un corpus compuesto por veinte artículos de cuatro revistas colombianas indexadas del área de lingüística aplicada.

En dieciocho artículos, la revisión teórica propia del MT se desarrolló bajo un título genérico (marco teórico, marco referencial, marcoconceptual, conceptualización, referente teórico, referencias teóricas), o bajo otros títulos alusivos a algunos conceptos claves de la investigación reportada. Cabe señalar que en dos artículos el MT se presentó de manera diferente: en uno, se incluyó en la introducción, y en el otro, se desarrolló desde el inicio, reemplazando así lo que comúnmente se denomina introducción.

Para los análisis, primero procedimos a identificar los procesos verbales presentes en los marcos teóricos. Después, identificamos a los participantes de la cláusula junto con sus proyecciones, para luego analizar el COMPROMISO, la ACTITUD y la GRADUACIÓn del sistema de la VALORACIÓN. En este artículo daremos cuenta del análisis del subsistema de GRADUACión, y más específicamente, de la FUERZA, siguiendo las categorías propuestas por Hood (2010) y Martin y White (2005). El corpus se dividió en cuatro grupos de cinco artículos cada uno; cada grupo de artículos fue asignado inicialmente a dos investigadores, quienes los analizaron por separado; luego, los acuerdos alcanzados por los dos investigadores se socializaron y discutieron con el grupo de investigadores en pleno (seis investigadores). Esto con la finalidad de darle mayor confiabilidad y validez al proceso de análisis.

En un principio, centramos el análisis en la cláusula verbal, pero luego nos percatamos de que era necesario considerar no solo la cláusula, sino también 
el párrafo o párrafos asociados, e incluso unidades discursivas mayores para tener en cuenta la prosodia, la propagación, la retropropagación y el acoplamiento (Hood, 2010). El proceso de análisis no fue lineal; por el contrario, varias veces tuvimos que volver a los análisis ya realizados debido a dificultades que se nos presentaban durante el proceso. Una de esas dificultades consistió en asumir que las categorías de VALORACión eran excluyentes. Al clasificar algunos datos en términos de FUERZA, nos percatamos de la superposición de esta con [heteroglosia: contracción: refuerzo: pronunciar] y [heteroglosia: contracción: refuerzo: justificar]. Por ello, en una última revisión de los análisis, decidimos clasificar estos casos como instancia de [compromiso: heteroglosia] y no como [graduación: fuerza], algo sobre lo que volveremos en la sección de resultados. Para los respectivos análisis, empleamos el software UAM Corpus Tool (O’Donnell, 2008).

\section{Resultados y discusión}

La Tabla 2 ilustra la distribución de los procesos verbales en el corpus analizado, incluidos los años de publicación de los textos analizados. Es notable la tendencia al aumento del promedio de procesos verbales por año con un pico en 2011. En adelante presentaremos inicialmente los recursos para la representación de la FUERZA en el corpus estudiado, luego su función y, finalmente, su despliegue en el MT.

\section{Recursos para la representación de la fuerza}

A continuación, ilustraremos los diversos recursos utilizados para intensificar y cuantificar la fuerza en el corpus estudiado.

\section{Intensificación infundida en el proceso}

La intensificación de una cláusula se puede dar por infusión de la FUERZA en el proceso mismo o por medios periféricos. La intensificación por infusión ocurre cuando una circunstancia de manera o modo está incluida en el proceso verbal, de forma que este adquiere mayor intensidad
Tabla 2 Distribución de los procesos verbales

\begin{tabular}{cccc}
\hline $\begin{array}{c}\text { Año de } \\
\text { publicación }\end{array}$ & $\begin{array}{c}\text { Número de } \\
\text { revistas }\end{array}$ & $\begin{array}{c}\text { Número de } \\
\text { cláusulas } \\
\text { verbales }\end{array}$ & $\begin{array}{c}\text { Promedio } \\
\text { de cláusulas } \\
\text { por año }\end{array}$ \\
\hline 2008 & 1 & 18 & 18 \\
2009 & 3 & 25 & 8,3 \\
2010 & 3 & 40 & 13 \\
2011 & 4 & 108 & 27 \\
2012 & 2 & 28 & 14 \\
2013 & 7 & 134 & 19 \\
\hline
\end{tabular}

(Hood, 2010, p. 92) y la irradia al informe o a la proyección. Ejemplos: ${ }^{2}$

(1) Esta teoría enfatiza la influencia de los contextos sociales y culturales en el conocimiento. (14.166.1)

(2) lo que Searle quiere destacar es que el lenguaje en los hechos institucionales no es solo descriptivo (19.192.1)

(3) En tal sentido, Bunton (2005) muestra que hay diferencias cualitativas entre la "Conclusión" como capítulo final de tesis, y las conclusiones como parte final del capítulo "Discusión". (10.158.2)

Los procesos 'enfatizar' y 'destacar' dan un realce adicional al contenido de la teoría (1), y a lo dicho por Searle (2); por su parte, 'muestra' (3), entendido como sinónimo de 'demuestra', puede indicar mayor grado de convicción con respecto a la proyección.

\section{Intensificación de la cláusula vía modificador del proceso}

También identificamos casos en los que la intensificación no está infundida en el proceso, sino que se realiza mediante otros recursos semánticos que funcionan como modificadores del proceso.

(4) Hatim y Mason (1997) son insistentes en afirmar que los géneros son elementos intersemióticos (4.438.4)

(5) Algunos autores se muestran partidarios de la replicación del CI y lo dicen explícitamente (6.24.3).

2 Entre paréntesis se indica el número del artículo, luego la página y, por último, el párrafo. Así, (14.166.1) significa que el ejemplo fue tomado del texto 14 del corpus, página 166 , párrafo 1 . 
En (4), el significado ideacional 'afirmar' recibe un significado actitudinal de juicio positivo de tenacidad 'insistentes' - referido a los autores Hatim y Mason-, lo que intensifica el proceso y extiende la FUERZA a la cláusula proyectada, por acoplamiento. En (5) la aseveración 'algunos autores se muestran partidarios de la replicación del CI’ se ve intensificada mediante dos estrategias: reiterando la aseveración con el proceso verbal 'dicen' y el informe 'lo', y mediante el adverbio 'explícitamente'.

\section{Intensificación vía cualidades}

En Martin y White (2005), la intensificación de cualidad se da mediante el uso de modificadores (adverbios o adjetivos) de un adjetivo dentro del informe. En el corpus analizado, cualidades con FUERZA infundida imprimen tanto la fuerza como la ACTITUD positiva a toda la cláusula, como en el siguiente ejemplo:

(6) El trabajo de Valarino da cuenta de importantes factores asociados con este problema, pero los relaciona exclusivamente con el tesista [...] (11.96.4)

En(6) el Informe de la cláusula verbal es expresado por un grupo nominal conformado por un premodificador (una cualidad) y un núcleo con posmodificadores. La intensificación está expresada mediante la combinación de una cualidad con FUERZA infundida y la posición marcada del adjetivo antes del sustantivo. En español, el adjetivo posmodificador se encuentra en posición no marcada; la posición premodificadora es, por consiguiente, marcada; tiene un carácter más subjetivo, y se extiende a todo el grupo nominal y no solamente al núcleo (Asociación de Academias de la Lengua Española, 2011).

\section{Cuantificación de la entidad}

Una forma de cuantificar la FUERZA de la cláusula es mediante el listado de autores o la repetición de un mismo autor a lo largo del texto. El listado de fuentes aporta FUERZA a la proyección o al Informe de la cláusula, con lo cual se logra una argumentación consistente que da mayor validez a la aseveración; por ejemplo:
(7) Los estudios de Ladino y Tovar (2005), Ochoa y Aragón (2004), y Rivera (2003) reconocen que los estudiantes no poseen estrategias estructuradas para enfrentarse a la tarea de leer un texto científico (5.538.4)

El hacer referencia a varios autores con sus respectivas investigaciones aumenta el valor de lo afirmado en 'los estudiantes no poseen estrategias'. Así, este recurso contribuye a dos de los propósitos del MT: posicionar al escritor como conocedor de otros miembros de su comunidad y de sus aportes al tema tratado, y al mismo tiempo, dar solidez a su propia afirmación, ya que varios autores la han confirmado.

El caso de la repetición de un mismo autor a lo largo del texto coincide con la contracción de la heteroglosia. Este recurso pone en evidencia la alineación del escritor con un autor que considera relevante en el tema. Tal es el caso de Searle en el ejemplo (8):

(8) [...] continuada por colaboradores cercanos como Searle $[\ldots](18.21 .2)$

[...] Searle posteriormente encontró algunas fallas en la taxonomía [...] (18.21.2)

[...] La definición que Searle da a los actos de habla (18.21.2)

Así mismo, Searle se encargó de enfatizar [...] (18.21.3)

Cabe destacar que Searle fue el primero en reconocer $[\ldots](18.21 .3)$

\section{Cuantificación del alcance del proceso}

En estos casos, la FUERZA aumenta o disminuye la validez de las afirmaciones, mediante la alusión a la cantidad de espacio que se dedica en el texto a una aseveración (9), la extensión geográfica en donde se ha validado la aseveración (10) o el lapso de tiempo durante el cual ha estado vigente la aseveración (11):

(9) A continuación se comentará brevemente qué aspectos se incluyeron tanto en la valoración inicial como en la valoración final de las habilidades de escritura del estudiante. (7.44.1)

(10) Estudios realizados en Colombia, Venezuela, Chile, Argentina, Brasil y Ecuador muestran que los índices de lectura en la población han disminuido drásticamente en los últimos años (5.538.3). 
(11) [N]uestro análisis, desde el punto de vista neurobiológico está fundamentado en el modelo propuesto por Kuhl (2005). De acuerdo con esta autora, el patrón emergente de la investigación neurobiológica sobre la adquisición del lenguaje es muy diferente del que se había sostenido históricamente. Los infantes no son tabula rasa como sostenía Skinner, ni 'lingüistas innatos' como formulaba Chomsky (13.212.4).

En (11) el modelo teórico escogido para el estudio del artículo trece se expresa con FUERZA mediante la oposición 'muy diferente' al punto de vista tradicional que había subsistido 'históricamente' [cuantificación: alcance: tiempo] y la negación rotunda a las afirmaciones de dos importantes figuras de la disciplina como son Skinner y Chomsky.

Igualmente, el aumento de FUERZA en el proceso puede ocurrir cuando se cuantifica la entidad, ya sea enumerando sus tipos, partes, pasos, entre otros; por ejemplo:

(12) Josefa Dorta Luis y María Noemí Domínguez García [...] en su artículo [...] proponen tres funciones discursivas [.... A) función organizadora [...] B) Función conectora [...] C) Función reformuladora [...] (15.30.3)

\section{Intensificación y cuantificación combinadas}

Una combinación de recursos de varias ramas del sistema confirma que no son categorías excluyentes:

(13) Varios estudiosos han señalado la importancia de la concepción de la norma de la cortesía verbal establecida en la sociedad al enfrentarse a la interacción (Noblia, 2001; Placencia, 2001), sobre todo de otras culturas (Bravo, 1999; Haverkate, 2000, 2004; Spencer-Oatey, 2000) [...] (2.50.1)

(14) Se han hecho hasta el momento tres importantes afirmaciones: la primera, [...] (20.310.2)

En el ejemplo (13), 'varios estudiosos' junto con los autores citados entre paréntesis, conforman cuantificación: entidad; además, la nominalización (importancia) imprime una intensificación de la FUERZA a la concepción de la norma de la cortesía verbal. De manera similar, en el ejemplo (14) el proceso 'se han hecho afirmaciones' se percibe con fuerza por la cuantificación de las partes del todo (tres afirmaciones) y la intensificación que imprime el epíteto 'importante', todo lo cual se irradia hacia la proyección directa que prosigue.

\section{Construcción prosódica de la fuerza}

A medida que desarrollan el texto, los escritores emplean una variedad de recursos retóricos para imprimir fuerza a sus afirmaciones, construir adhesiones a algunos autores y, así, imprimir validez a su marco teórico. Para ello recurren a cláusulas verbales que propagan la FUERZA mediante dominación - prospectiva o retrospectiva - irrigando fuerza hacia lo que se desarrolla más adelante o lo que se discutió líneas atrás.

La prosodia de intensificación se manifiesta con opciones que están altamente cargadas de ACTITUD (Hood, 2010, p.159). La expresión de una ACTITUD positiva hacia un autor-emisor se puede propagar en un segmento discursivo, mediante acoplamiento desde las expresiones de ACTITUD positiva hacia el proceso verbal y su proyección. Tal es el caso que se ilustra en el ejemplo (15), en donde Searle es 'ampliamente conocido', y su llamado a considerar 'las asunciones que subyacen al sentido común del mundo científico' es 'particularmente relevante'. De allí que, en estas cláusulas, lo afirmado por Searle adquiere ACTITUD positiva por propagación. Así, el acoplamiento y la dominación escalan la FUERZA de la teoría de Searle en este fragmento, como una manera del escritor de fundamentar su alineación conceptual en su MT.

(15) Para establecer la relación entre el lenguaje y estos dos procesos psicológicos sugeridos por Moscovici, son de utilidad algunas reflexiones provenientes de la filosofía de la mente. El filósofo John Searle, ampliamente conocido en los estudios del lenguaje por su teoría de los actos de habla, afirma que debemos considerar, primero que todo, las asunciones que subyacen al sentido común del mundo científico. Esta invitación resulta particularmente relevante cuando se trata de analizar desde nuestro sentido común de científicos, las representaciones sociales que conforman el sentido común de aquellos que formulan las políticas educativas objeto de nuestros análisis.

La visión del mundo de la ciencia, dice Searle (1995), depende de [...] A la luz de estas distinciones, este 
filósofo sostiene que los rasgos estructurales de nuestro mundo [...] Desde esta perspectiva, Searle concluye que lo único que hay, en últimas es conocimiento y que la 'ciencia' es "un nombre que nosotros aplicamos a áreas en las que el conocimiento ha llegado a ser sistemático" (Searle, 1995, p.151) [...]

Searle afirma que la realidad social se refiere [...]; sin embargo, es tan real como una piedra, dice este filósofo, porque la realidad social ha sido creada por nosotros para nuestros fines y es tan inteligible como nuestros fines mismos [...] por esta razón, dice.

Continúa Searle afirmando que una subclase especial de los hechos sociales la conforma Searle afirma que el lenguaje constituye parcialmente los hechos institucionales porque $[\ldots]$

El principio que regula la creación inicial de un hecho institucional lo formula Searle de la siguiente manera: $[\ldots]$

Con ello, lo que Searle quiere destacar es que el lenguaje en los hechos institucionales no es solo descriptivo, sino que constituye la realidad social misma. Al relacionar la teoría de las representaciones sociales de Moscovici con la teoría de la construcción de la realidad social de Searle, puede concluirse que las representaciones sociales son conocimientos compartidos y justificados por un grupo de seres humanos acerca de hechos del mundo que existen en tanto este grupo de personas creen en y están de acuerdo sobre ellos. (19.189-193)

\section{Funciones de la fuerza}

A continuación se ilustran las funciones de la FUERZA para realzar las afirmaciones propias, las de otros autores y para organizar el discurso.

\section{Fuerza en las afirmaciones propias}

Los resultados de nuestros análisis nos permitieron establecer que, en el MT de un artículo de investigación de la lingüística aplicada, la FUERZA se puede construir con recursos no solo del subsistema de GRADUACIÓN sino también con los del subsistema de COMPROMiso, específicamente como [heteroglosia: contracción: refuerzo: pronunciar] cuando los escritores quieren dar realce a su propia voz autorial (ejemplos 16 y 17), o [COMPROMISO: heteroglosia: contracción: refuerzo: justificar] cuando se desea irradiar FUERZA hacia las voces de otros autores (ejemplo 18).
(16) Es conveniente hacer dos anotaciones (1.31.1)

(17) Por último, nos parece importante destacar nuevamente que es, sin duda, el escenario comunicativo virtual el que determina el alcance de los actos de habla ilocutivos no literales (18.24.1).

En el corpus analizado, las instancias de [heteroglosia: contracción: declaración: refuerzo: pronunciar], como (16) y (17), son construidas con expresiones de modulación como 'es conveniente,' 'se hace necesario señalar', 'cabe destacar', 'es importante hacer notar', 'conviene aclarar', 'es necesario referirse', 'es preciso enfatizar', mediante las cuales el escritor gradúa la intensidad de sus propias aseveraciones.

Para construir la FUERZA en su voz mediante las afirmaciones de otros autores, los escritores utilizan [heteroglosia: contracción: refuerzo: justificar] (White, comunicación personal)

(18) $[\ldots]$ es necesario realizar inferencias, ya que, como lo afirma García (1993), el texto no proporciona explícitamente toda la información requerida (5:535:4) (19) Sin embargo, no es ésta la manera como la universidad asume la escritura, quizás porque, como dice Cassany (2003), no existe una [...] del lenguaje verbal (16.319.3)

En el ejemplo (18) el escritor le imprime mayor valor a su aseveración acerca de la necesidad de realizar inferencias, incorporando la voz de un autor (García) mediante una cláusula verbal, cuya proyección se constituye en la justificación o razón de la afirmación inicial. En el ejemplo (19), el escritor sustenta la negación de la forma como la universidad asume la escritura y construye la FUERZA, recurriendo a la voz de Cassany.

\section{Fuerza a las afirmaciones de autores sustento del marco teórico}

La autoridad del escritor en gran medida se construye dando validez a la teoría escogida. La fuerza que se imprime a los conceptos, nociones o ideas que sirven de sustento al estudio propio se expresa intensificando las aseveraciones de los autores referenciados al explicitar la fuente o la lógica de las afirmaciones. 
(20) En este sentido, Serrano (1996), partiendo de las afirmaciones de Genette y Benveniste, sostiene que el texto narrativo se fundamente sobre una base verbal [...] (20.311.4)

(21) La escritura, por ejemplo, dice Ortiz, surgió como una necesidad específica de la sociedad occidental "racional" para poder expresar la universalidad del pensamiento occidental. Esta articulación del discurso sobre la diferencia a (sic) intereses histórica y socialmente determinados lleva a concluir a Ortiz que el debate sobre la diversidad cultural tiene implicaciones políticas. (19.195.1)

Los escritores en (20) y (21) utilizan una síntesis de un antecedente (en subrayado) que sirve como fundamentación para sustentar conclusiones de autores citados en el MT. De esta manera, el MT valora la pertinencia de un razonamiento previo para justificar una acertada elección de autores y conceptos en su propio marco conceptual. $\mathrm{Al}$ expresar FUERZA, los escritores agregan significados que son una interpretación de los originales. En (22) con el proceso verbal 'destaca', el escritor juzga e intensifica lo afirmado por los autores escogidos para el MT.

(22) Peters y Carlsen (1991) destacan [...] (20.305.3)

\section{Distribución de la fuerza y función textual}

En el MT identificamos cláusulas verbales con FUERZA en secciones organizadoras de los textos, como son los macroTemas, hiperTemas, hiperNuevos y macroNuevo. Es posible, entonces, que la fuerza contribuya a la función organizadora de estos segmentos.

Los siguientes casos se identificaron en el macroTema de sus respectivos marcos teóricos.

(23) Haremos referencia a las tres dimensiones del contexto (4.437.4) [fuerza: cuantificación: entidad]

(24) El estudio de la literatura académica que orienta esta investigación, nos permitió recoger asuntos que [...] En este sentido, hacemos acopio de tres conceptos macro: formación en autonomía, constructivismo social y enfoque por competencias orientado a la acción (14.164.1) [fuerza: cuantificación: entidad]

(25) Desde esta visión, el presente estudio es significativo, puesto que se trata de mostrar algunos aspectos importantes de fórmulas de tratamiento por medio de las cuales, como se muestra a continuación, se formula expresamente la cortesía o la descortesía, sobre todo en Medellín, donde no se ha estudiado suficientemente este fenómeno, siempre cambiante a través del tiempo. (2.50.2) [Fuerza: cuantificación: entidad]

Los tres ejemplos anteriores, cuya función es evidentemente catafórica, se encuentran en sendos macroTemas del MT. En esta posición, las 'tres dimensiones del contexto' en (23) y 'los tres conceptos macro' en (24) que se desarrollan en el cuerpo del MT, cuantifican los conceptos claves enfatizando sus varios componentes; en (25) la cualidad de 'importante' se propaga al resto del desarrollo de las fórmulas de tratamiento que constituyen el marco conceptual.

Esta misma función catafórica se evidenció en la posición de hiperTema. Tal es el caso del ejemplo (26), en el cual la FUERZA se construye mediante la intensificación de la cualidad del Informe.

(26) Ahora bien, en relación con los tipos de cambios semánticos, son múltiples las clasificaciones de dichas posibilidades, pero la propuesta que elabora el profesor Montes (1983) para analizar la motivación y creación léxica en el español de Colombia resulta pertinente, porque, además de ser exhaustiva, está pensada para un léxico, en cierto modo, familiar al argótico, al menos en lo que respecta al idioma y al país. (T.12)

Aquí, el escritor está justificando la selección de una propuesta teórica, que en la cláusula verbal se resume en el Informe como 'la propuesta'. Este ángulo teórico se califica como 'pertinente', 'exhaustivo' y 'familiar' al léxico argótico, lo cual es importante para un estudio que se centra en el argot de las reclusas en una cárcel colombiana. Esta construcción en posición de hiperTema propaga la FUERZA en la fase, cumpliendo simultáneamente funciones interpersonal y textual (Hood, 2010). La FUERzA de las cláusulas verbales se puede propagar hacia atrás, para reforzar conceptos expuestos antes, como se ilustra en el siguiente ejemplo:

(27) Para resumir, Hatim y Mason (1997) son insistentes en afirmar (4.438.4) 
Veamos la distribución de la fuerza en las cláusulas verbales en los puntos prominentes del MT del texto (15). Para ilustrar mejor la forma como se despliega la información por niveles jerárquicos, los macroTemas y los macroNuevos ocupan el espacio de un renglón completo, mientras que los hiperTemas e hiperNuevos, que corresponden a un nivel inferior, se presentan alineados un poco más hacia la derecha, y los procesos dentro del desarrollo de su respectiva fase se alinean todos también hacia la derecha, pero con un margen mayor con respecto a los hiper-y macroTemas.

\section{(28) MacroTema}

En este apartado se recogen las definiciones que han dado varios autores de las diferentes funciones de pues $[\ldots]$

De igual manera (La Real Academia Española) señala [...] Cabe anotar [...]

Así mismo, en una de sus acepciones, el DRAE expone (29) HiperNuevo

Se puede ver que en la definición que propone la Real Academia Española de la unidad pues se señalan sus funciones

(30) HiperTema

Por otra parte, Portolés [...] propone distribuir los usos de la unidad pues en tres grupos...

Marroquín y Portolés señalan [...]

De igual manera, estos autores resaltan...

Además, agregan [...]

Portolés advierte

(31) HiperNuevo

Este autor concluye que el pues comentador no se acomoda perfectamente dentro de las categorías de las conjunciones

(32) HiperTema

Otra propuesta de definición de las funciones de pues es la elaborada por Josefa Dorta Luis, María Noemí Domínguez García. En su artículo [...] proponen tres funciones discursivas $[. .$.

(33) HiperNuevo

Al comparar la función organizadora de marcador del discurso pues propuesta por Dorta y Domínguez con la función de pues comentador en la clasificación de Portolés puede corroborarse que se corresponden, pues las dos definiciones señalan que se presenta introduciendo un nuevo aspecto del discurso [...]

(34) HiperTema

María del Rosario Uribe Mallarino [...] se basa en la clasificación [...] propuesta por Briz

(35) HiperNuevo
En la clasificación de Portolés no aparece la función metadiscursiva [...] pero los resultados de la investigación de Uribe Mallarino (2006), en los que muestra que en el corpus que analizó pues cumple estas funciones, amplían las posibilidades de este marcador

(36) HiperTema

Catherine Travis [...] define siete funciones para [el marcador pues]

(37) HiperNuevo

Como se puede observar, las funciones de adición de información, así como [...] las funciones de focalización y marca de cierre del tema no habían sido definidas por los otros autores

(38) MacroNuevo

De acuerdo con las clasificaciones de las funciones del marcador discursivo pues que hemos presentado en este apartado, se puede afirmar que este marcador discursivo puede cumplir múltiples funciones: conjunción, comentador, conector consecutivo, reformulador metadiscursivo, marcador de control de contacto focalizador y marcador de cierre de tema.

En los ejemplos (28) a (38), las cláusulas verbales ayudan a cumplir la función textual, con lo cual dan organización y jerarquía a la información en el texto. En todos los segmentos marcados como macroTema, hiperTema, hiperNuevo y macroNuevo hay cláusulas verbales. Los picos de información presentados en el macroTema preparan al lector con respecto a la información que se presentará más adelante, en este caso, las funciones del marcador 'pues'. Estas se despliegan a lo largo del texto, con picos un poco menos protuberantes en los hiperTemas y los hiperNuevos. En los hiperTemas de (30), (32), (36) se cuantifica la FUERZA al anunciar las funciones y usos del marcador 'pues' (los usos de la unidad 'pues' en tres grupos, proponen tres funciones discursivas, definen siete funciones).

Por otro lado, el uso de cláusulas verbales en los hiperNuevos retoma la centralidad del emisor, que en estos casos son los autores que aportan las definiciones de las funciones. Además, en (31), el escritor recurre a la cláusula verbal [heteroglosia: contracción: declaración: justificación] para argumentar su aceptación a la función del marcador 'pues' propuesta por Portolés, Dorta y Domínguez. Con ello, el escritor va construyendo la conclusión que presenta en el macroNuevo. Allí (38), el 
escritor recoge todas las definiciones de funciones del marcador 'pues' hacia las que ha mostrado una ACTITUD positiva y que, por tanto, acepta y adopta para su estudio.

\section{Conclusiones}

En el мт de los artículos de investigación, las cláusulas verbales juegan un papel importante, porque dan cuenta de la posición del escritor y de las diferentes voces que han sido escogidas para servir de marco o sustentar una investigación, ya sea para alinearse con ellas o para tomar distancia. Ese posicionamiento se construye en gran medida mediante recursos del sistema de la VALORACIÓN, aunque aquí nos hemos referido específicamente a los del subsistema de la GRADUACIÓn. En el corpus escogido hemos examinado cómo, mediante cláusulas verbales, los escritores aumentan la FUERZA en sus actitudes positivas hacia las propias aseveraciones y en las de los autores que ellos citan.

88 El uso de estas cláusulas verbales hace visible al conocedor, tal vez más que el conocimiento mismo (Hood, 2011; Maton, 2009). Esto no sucedería si los escritores usaran otro tipo de cláusula y dieran a conocer los autores entre paréntesis o como complementos de ángulo. Dado el promedio de cláusulas verbales por artículo (18), se podría afirmar que, en el área de lenguaje, los investigadores que publican en español tienen la tendencia a colocar a sus autores-referentes en lugar visible y prominente, en la cresta de las "oleadas de información” (Martin y Rose, 2007), con lo cual enfatizan la fuente del Informe o la Proyección. La posición estratégica de las cláusulas verbales en macroTemas, hiperTemas, hiperNuevos y macroNuevos construye la FUERZA de formas que tienen alcance más allá de las fronteras de la cláusula verbal, contribuyendo al fenómeno de propagación de prosodias evaluativas, mediante mecanismos de dominación. El uso de los recursos que aumentan la ACTITUD hacia ciertos referentes teóricos construye a un escritor consciente y muy comprometido con su marco conceptual, decidido a ganar la aprobación y la alineación de su lector con los referentes teóricos valorados por él (Martin y White, 2005).

Las cláusulas verbales resultan centrales para el desarrollo de las etapas del MT descritas por Tseng (2018), en especial, para implementar estrategias como las de anunciar la adopción de una perspectiva teórica particular, introducir un trabajo clave dentro de la propuesta conceptual escogida, mostrar de manera organizada las partes de las que consta el constructo, justificar su selección, recoger y enfocar las afirmaciones teóricas que son significativas para el estudio.

En este sentido, enseñar a escritores noveles a construir la voz autorial mediante el uso consciente de cláusulas verbales en el MT resulta relevante toda vez que posibilita su posicionamiento en la sociedad del conocimiento de la que desean formar parte. Asimismo, el manejo apropiado de los recursos para construir la FUERZA posibilita a los escritores noveles no solo atribuir la información a una determinada fuente, sino valorar su pertinencia, la importancia y el realce de los argumentos que servirán de soporte a su planteamiento. Así, el escalamiento de la evaluación mediante la FUERZA permite dialogar con la objetividad que se espera en esta sección del artículo de investigación.

Reconocemos, sin embargo, que estas consideraciones son un primer acercamiento a los recursos de VALORACIÓN en cláusulas verbales en el contexto del marco teórico, puesto que esta sección sigue siendo una de las menos estudiadas. Quedan así caminos por explorar: cómo se realiza la FUERZA en los marcos teóricos que se construyen sin procesos verbales, cómo se percibe la voz autorial sin ellos, cómo contrasta el uso de cláusulas verbales en español con respecto a otras lenguas y en otras disciplinas.

\section{Referencias}

Asociación de Academias de la Lengua Española. (2011). Nueva gramática de la lengua española. Manual. Madrid: España. Recuperado de http://www.ceip.edu. uy/IFS/documentos/2015/lengua/recursos/gramatica_raenueva.pdf 
Bazerman, C. (1988). Shaping written knowledge. Madison: University of Wisconsin Press.

Beangstrom, T. y Adenorff, R. (2013). An appraisal analysis of the language of real estate advertisements. Southern African Linguistics and Applied Language Studies, 31(3), 325-347. https://doi.org/10.2989/1 6073614.2013 .837608

Chatterjee-Padmanabhan, M. (2011). Identity and textual engagement: Experiences of three international students writing a doctoral thesis in EAL. Doctor of Education Thesis. Faculty of Education. University of Wollongong, Australia.

Cisneros-Estupiñán, M. y Muñoz, D. (2014). Los mecanismos de la valoración en la construcción del discurso en el aula universitaria. Revista Colombiana de Educación, 66, 247-263. https://doi.org/10.17227/012 $03916.66 \mathrm{rce} 245.262$

Cunillera-Domènech, M. y Andújar-Moreno, G. (2017). La expresión lingüística de la valoración en textos jurisprudenciales: Estudio contrastivo francés-español. Revista Signos. Estudios de lingüistica, 50(94) 174-194. https://doi.org/10.4067/ S0718-09342017000200174

Derewianka, B. (2007). Using appraisal theory to track interpersonal development in adolescent student writing. En A. McCabe, M. O 'Donnell y R. Whittaker (Eds.), Advances in language and education (pp.142-165). Nueva York: Continuum.

Halliday, M. A. K. (1979). Modes of meaning and modes of expression: Types of grammatical structure, and their determination by different semantic functions. En D. J. Allerton, E. Carney y D. Holdcroft (Eds.), Function and context in linguistic analysis (pp. 57-79). Cambridge: Cambridge University Press.

Halliday, M. A. K. y Matthiessen, C. M. I. M. (1999). Construing experience through meaning: A languagebased approach to cognition. Londres: Cassell.

Halliday, M. A. K. y Matthiessen, C. M. I. M. (2014). Halliday's introduction to functional grammar ( $4^{\mathrm{a}}$ Ed.). Londres: Routledge. https://doi. org/10.4324/9780203783771

Herrero Rivas, L. E. (2013). Transitividad y procesos verbales en el lenguaje académico en español: Una perspectiva sistémico-funcional. Lenguas en contexto, 10, 36-44.

Hood, S. (2006). The persuasive power of prosodies: radiating values in academic writing. Journal of English for Academic Purposes, 5(1), 37-49. https://doi. org/10.1016/j.jeap.2005.11.001

Hood, S. (2010). Appraising research: Evaluation in academic writing. Londres: Palgrave. https://doi. org/10.1057/9780230274662

Hood, S. (2011). Writing discipline: comparing inscriptions of knowledge and knowers in academic writing. En F. Christie y K. Maton (Eds.), Disciplinarity: Functional linguistic and sociological perspectives (pp. 106-128). Londres: Continuum.

Hood, S. y Martin, J. (2005). Invoking attitude: The play of graduation in appraising discourse. Revista $\mathrm{Si}$ gnos, 38(58),195-220. https://doi.org/10.4067/ S0718-09342005000200004

Ignatieva, N. (2011). Procesos verbales en la escritura académica estudiantil en español desde una perspectiva sistémico funcional. Lenguaje, 39(2), 447-467. https://doi.org/10.25100/lenguaje.v39i2.4939

Ignatieva, N. y Rodríguez-Vergara, D. (2015). Verbal processes in academic language in Spanish: Exploring discourse genres within the systemic functional framework. Functional Linguistics, 2, 2-14. https:// doi.org/10.1186/s40554-015-0014-9

Kaplan, N. (2004). Nuevos desarrollos en el estudio de la evaluación en el lenguaje: La teoría de la valoración. Boletín de Lingüistica, 22, 52-78 [en línea]. Disponible en: http://www.redalyc.org/articulo. oa?id=34702203

Lemke, J. (1998). Resources for attitudinal meaning: Evaluative orientation in text semantics. Functions of Language, 5, 33-56. https://doi.org/10.1075/ fol.5.1.03lem

Love, K. y Arkoudis, S. (2006). Teachers'stances towards Chinese international students: An Australian case study. Linguistics and Education, 17, 258-282. https://doi.org/10.1016/j.linged.2006.11.002

Marimón, C. (2014). Prácticas discursivas académicas en la economía: Aproximación crítica a las estrategias de valoración. Revista de Investigación Lingüistica, 17, 75-95.

Martin, J. R. (1992). English text: system and structure. Ámsterdam: John Benjamins. https://doi. org/10.1075/z.59

Martin, J. R. (2009). Boomer dreaming: The texture of recolonisation in a lifestyle magazine. En G. Forey y G. Thompson (Eds.), Text type and texture (pp. 252284). Londres: Equinox. 
Martin, J. R. (2010). Affiliation: Realization, instantiation and individuation in a Bostwanan town. En M. Bednarek y J. R. Martin (Eds.), New discourse on language: Functional perspectives on multimodality, identity and affiliation. Londres: Continuum.

Martin, J. R. y White, P. R. R. (2005). The language of evaluation: Appraisal in English. Basingstoke/ Nueva York: Palgrave Macmillan. https://doi. org/10.1057/9780230511910

Maton, K. (2007). Knowledge-knower structures in intellectual and educational fields. En F. Christie y J. Martin (Eds.), Language knowledge and pedagogy. Functional linguistic and sociological perspectives (pp. 87-108). Londres: Continuum.

Maton, K. (2009). Cumulative and segmented learning: Exploring the role of curriculum structures in knowledge-building. British Journal of Sociology of Education, 30(1), 43-57. https://doi. org/10.1080/01425690802514342

Matthiessen, C. M. I. M. (1995). Lexicogrammatical cartography. English systems. Tokio: International Language Sciences Publishers.

Moyano, E. (2010). Exploring verbal processes in discussions of research articles in Spanish. En C. Wu y Y. Fang (Eds.), Challenges to systemic functional linguistics: Theory and practice. Proceedings of the $36^{\text {th }}$ International Systemic Functional Congress. Pekín: $36^{\text {th }}$ ISFC Organizing Committee, Tsinghua University and Macquarie University. PRC, 13-18 de julio.

Navarro F. (2014) Gradación y compromiso en escritura académica estudiantil de humanidades. Análisis contrastivo desde la teoría de la valoración. Estudios de Lingüistica Aplicada, 60, 9-33.

O'Donnell, M. (2008). Demonstration of the UAM Corpus Tool for text and image annotation. En Proceedings of the ALC-08: HLT Demo Session (Companion Volume). Columbus, Ohio, 13-16 de junio. Stroudsburg, PA: Association for Computational Linguistics. https:// doi.org/10.3115/1564144.1564148

Oteíza T.y Pinuer, C. (2010). La temporalidad, recurso estratégico en documentos oficiales de derechos humanos en Chile. Estudios Filológicos, 46, 81-99. https://doi. org/10.4067/S0071-17132010000200005

Pascual, M. (2014). La asociación de recursos de valoración: El caso de las organizaciones de derechos humanos en la prensa escrita argentina. Onomázein, número especial ALSFAL, 99-114. https://doi.org/10.7764/ onomazein.alsfal.8

Pho, P. D. (2013). Authorial stance in research articles: Examples from applied linguistics and educational technology. UK: Palgrave Macmillan. https://doi. org/10.1057/9781137032782

Rose, D. y Martin, J. (2012). Learning to write, reading to learn. Genre, knowledge and pedagogy in the Sydney School. Sheffield: Equinox.

Santosa, R., Priyanto, A. D. y Nuraeni, A. (2014). Genre and register of antagonist's language in media: An appraisal study of Indonesian newspapers. Surabaya, 16(1), 23-36.

Serpa, C. (2013). Significados de probabilidad y fuerza en los textos legislativos. I Vardande. Revista Electrónica de Semiótica y Fenomenología Jurídicas, 1(1), 3-33.

Swales, J. (1990). Genre analysis: English in academic and research settings. Cambridge: Cambridge University Press.

Swales, J. (2004). Research genres: Explorations and applications. Cambridge: Cambridge University Press. https://doi. org/10.1017/CBO9781139524827

Swales, J.y Lindemann, S. (2002) Teaching the literature review to international graduate students. En A. M. Johns (Ed.), Genre in the classroom: Multiple perspectives (pp. 105-20). Mahwah, NJ: Lawrence Erlbaum.

Sutherland, A. y Adendorff, R. (2014). An appraisal analysis of a Women's Day rant, rally and reflection. Southern African Lingusitics and Applied Language Studies, 23(4), 393-415. https://doi.org/10.2989/16073614.2014.9 97070

Thompson, G. (1998). Resonance in text. En A. SánchezMararro y R. Carter (Eds.), Linguistic choice across genres: Variation in spoken and written English (pp. 2963). Ámsterdam: John Benjamins. https://doi. org/10.1075/cilt.158.05tho

Tseng, M. (2018). Creating a theoretical framework: On the move structure of theoretical framework sections in research articles related to language and linguistics. Journal of English for Academic Purposes, 33, 82-99. https://doi.org/10.1016/j.jeap.2018.01.002

Yang, R. y Allison, D. (2003). Research articles in applied linguistics: Moving from results to conclusion. English for Specific Purposes, 22(4), 365-85. https://doi. org/10.1016/S0889-4906(02)00026-1

Xiaoyu, X. (2017). An analysis of stance and voice in research articles across Chinese and British cultures using the appraisal framework. Doctoral dissertation, Coventry University, Gran Bretaña.

Zappavigna, M. (2011). Ambient affiliation: A linguistic perspective on Twitter.New MediaySociety, 13(5),788806. https://doi.org/10.1177/1461444810385097 
How to reference this article: Barletta, Norma Patricia; Chamorro, Diana; Mizuno, Jorge. (2020). La fuerza en la construcción del marco teórico en artículos científicos: un análisis desde la lingüística sistémica funcional. Íkala, Revista de Lenguaje y Cultura, 25(1), 75-91, http://www.doi.org/10.17533/ udea.ikala.v25n01a14 